Reprod. Nutr. Dévelop., 1988, 28 Suppl. n¹, 169-170

\title{
Expression hépatique du gène d'apolipoprotéine $B$ chez la vache au cours de la lactation
}

\section{P. CARDOT, A. MAZUR, Marcia PESSA $\left({ }^{*}\right)$, J. CHAMBAZ $\left({ }^{* *}\right)$, Y. RAYSSIGUIER}

Laboratoire des Maladies Métaboliques,

I.N.R.A., Theix, 63122 Ceyrat, France.

(*) Centre de Recherche INSERM.

C.H.U. Saint-Antoine, 75571 Paris Cedex 12.

${ }^{* *}$ UA. CNRS 524, Laboratoire de Biochimie.

Faculté de Médecine Saint-Antoine, 75571 Paris Cedex 12.

Summary. Variations in the apoprotein $B$ hepatic expression were evaluated during lactation in dairy cows. Apoprotein B mRNA levels were significantly decreased in early lactation and this was accompanied by triglycerides accumulation in the liver.

Bien que le taux plasmatique des lipoprotéines légères riches en triglycérides soit faible chez la vache laitière, cette fraction joue un rôle important comme source de lipides pour la glande mammaire (Moore et Christie, 1981). Or ce taux de lipoprotéines est abaissé chez la vache en début de lactation et plus particulièrement chez celle qui présente une stéatose hépatique (Mazur et al., 1988 ; Rayssiguier et al., 1988). Ce phénomène pourrait s'expliquer par une diminution de la synthèse et/ou de la sécrétion hépatocytaire des lipoprotéines de très basse densité (VLDL). La sécrétion des VLDL et donc des triglycérides qu'elles exportent, est très probablement régulée au niveau de la synthèse des apolipoprotéines, et plus particulièrement de l'apolipoprotéine B (apo B). Une approche moléculaire doit permettre une meilleure compréhension de cette régulation. Nous avons entrepris cette étude à l'aide d'une sonde d'ADN complémentaire d'apo $\mathrm{B}$ humaine et d'apo $\mathrm{B}$ de rat que nous avons clonée.

Matériel et méthodes. Une biopsie hépatique est effectuée 15 jours avant le vêlage, et 6 et 20 jours après, sur des vaches frisonne pie-noires, fortes productrices, d'une même exploitation. Les échantillons sont immédiatement congelées dans l'azote. Les ARN totaux sont extraits par le thiocyanate de guanidine (Chirgwin et al., 1979). Ils sont ensuite, soit séparés sur gel d'agarose $1 \%$ après dénaturation au glyoxal et transférés sur filtre de nitrocellulose, soit dénaturés en présence de formaldéhyde et déposés par dilutions successives de $1 / 2$ en $1 / 2$ sur filtre de nitrocellulose. Les hybridations ont été réalisées avec une sonde d'apo $B$ humaine et une sonde d'apo $B$ de rat que nous avons obtenue à partir d'une banque d'ADNc de foie de rat construite dans le vecteur lambda gt 11. Les filtres sont hybridés à $42^{\circ} \mathrm{C}$ pendant $16 \mathrm{~h}$ avec les sondes d'ADN complémentaire d'apo $B$ marquées au $\left(\alpha \mathrm{P}^{32}\right) \mathrm{dCTP}$ ( $3000 \mathrm{Ci} / \mathrm{mmoles}$ ) par nick translation (activité spécifique $10^{8} \mathrm{cpm} / \mu \mathrm{g}$ ), dans un tampon phosphate $20 \mathrm{mM}$, pH 6,5, contenant du SSC 5x, du Denhardts $5 x, 20 \%$ de formamide et $100 \mu \mathrm{g}$ d'ADN de sperme de hareng. Les filtres après hybridation sont rincés trois fois avec une solution contenant $2 \times \mathrm{SSC}, 0,1 \% \mathrm{NaDodSO}_{4}$ 
pendant 10 minutes à $42^{\circ} \mathrm{C}$, puis 1 heure à $45^{\circ} \mathrm{C}$ dans du $1 \times \mathrm{SSC}$, puis autoradiographiés. Les « dots " sont ensuite découpés et comptés au compteur à scintillation. La teneur en lipides plasmatiques et en triglycérides hépatiques a été déterminée par voie enzymatique (Mazur et al., 1988).

Résultats et discussion. Les sondes que nous avons utilisées permettent de mettre en évidence la présence d'ARNm d'apo $B$ dans le foie de bovin et sont donc des outils appropriés pour étudier les variations de l'expression du gène d'apo $B$ chez cette espèce.

$\mathrm{Au} 6^{\mathrm{e}}$ jour de la lactation, la synthèse d'ARNm d'apo $\mathrm{B}$ est inférieure d'environ $50 \%$ par rapport au tarissement et à la $3^{\mathrm{e}}$ semaine de lactation (fig. 1 ). Parallèlement, nous observons pour cette période une augmentation du taux de triglycérides hépatiques ainsi qu'une diminution du taux de lipides plasmatiques (fig. 1). Donc au tout début de lactation la capacité limitée du foie à produire des VLDL riches en triglycérides pourrait s'expliquer par une diminution du taux de synthèse d'apo $B$. L'expression du gène hépatique augmente au cours de la lactation pour atteindre le taux observé chez la vache tarie.

En conc/usion, il existe une relation inverse entre, d'une part, les lipides plasmatiques et le messager apo $B$ et, d'autre part, les triglycérides hépatiques. $C e$ résultat est à rapprocher des résultats observés chez le rat lors de la stéatose induite par la galactosamine (Mangeney, communication personnelle). L'utilisation de cette sonde doit permettre une nouvelle approche du métabolisme hépatique des lipoprotéines chez la vache.

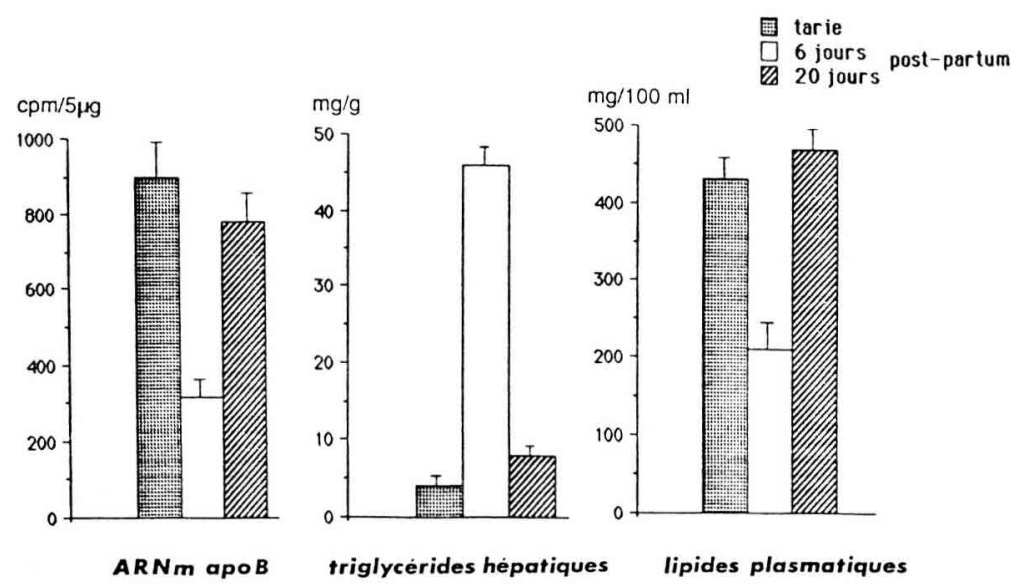

FIG. 1. - Variation du taux d'ARNm d'apolipoprotéine $B$, des triglycérides hépatiques et de la lipémie chez la vache avant vêlage et au cours de la lactation (les résultats sont la moyenne \pm S.E.M. de trois déterminations).

Chirgwin J. M., Przybyla A. E., Mac Donald R. J., Rutter W. J., 1979. Biochemistry, 18, 5294-5299. Mazur A., Gueux E., Chilliard Y., Rayssiguier Y., 1988. J. anim. Physiol. anim. Nutr., 59, $233-237$. Moore J. H., Christie W. W., 1981. In: Christie W. W., Lipid Metabolism in ruminant animals, Pergamon Press, pp. 227-277.

Rayssiguier Y., Mazur A., Reid I. M., Roberts C. J., Gueux E., 1988. Res. vet. Sci., 45, $389-393$. 